\title{
Eficacia de la Rifampicina en el tratamiento de los portadores de Meningococo.
}

\author{
Autores: J. Manuel Botgono, M.D., M.P.H.; Héctor Rodriguez, M.D., M.P.H.; Julio Garcia, M.D., e Irma Cánepa
} R.X.

\begin{abstract}
The role of carriers as well as current șhortcomings in thenrapentic schemes available, being main problems in latest outbreaks of meningocoxcal meningitis, have pointed out the need for research on new and effective prophylactic measures.

Eflectiveness and innocuity of Rifampicin (Rimactan) were tested by the authors in 2.132 children, a-ed from I to 18 years, attending kindergartens and elementary schuols in Santiago, Chile.

$12 \%$ were diagrosed as ineningocectal carriers by throkt swab; these carriers were given Rifampicin or placebo following selection throught aleatory sampling. Phanngeal tests were repeated on th 3rd. and 10 th day after treatment. While $92 \%$ of carriers unde Rifamplcin became negalive on the third day. 35\% of those carriers thetting placebo were negative on the sance date.

16\% of carriers whose pharyngeal test thecame negative on the 3 rd day under the Rifampicin schense, were still negative on the IO th day's test.

Symptoms ar elinical signs of secondany effects of Rifampicin were not found. Bifampicin resistant strains were not fuund.
\end{abstract}

Los problemas sanitarios presentados por las recientes epidemias de meningitis meningocóci. cas en varios países, junto a la importancia de los portadores de meningococo en estos brotes, indican la necesidad de contar con nuevas armas efectivas e inocuas para este control y tratamiento.

El presente trabajo esta dedicado a investigas la eficacia y la inocuidad de la Rifarnpicina (Rimac$\tan$ ) en el tratamiento de niños portadores de meningococo, incluyendo también la observación clínica para conocer los posibles efectos colaterales de la droga y el estudio de aparición de resistencia del meningococo a este antibiótico.

\section{MATERLAL, Y ME TODO}

Durante un periodo de trece meses, comprendido entre abril y noviembre de 1977, y junio a agosto de 1979 , se procedió a tomar muestras de secreción faríngea a 2.132 niños entre 2 y 15 años de edad, con el fin de identificar su condición de portadores de meningococo.

Estos niños provenían de jardines infantiles y escuelas de educación básica de la ciudad de Santiago de Chile, elegidos al azar, y empleando como unidad de muestreo un jard in o escuela.

El frotis faringeo fue realizado por una enfermera adiestrada, y la muestra fue procesada en e] Instituto de Salud Pública, de acuerdo con la técnica de aislamiento con medio de Thayer Martin, crecimiento de Müller Hinton e identificación por pruebas de azúcar y serología. La determina- ción de la sensibilidad del meningococo ante la Rifampicina se efectuó mediante la técnica de Kirby Bauer con difusión.

Aquellas personas individualizadas como portadoras de meningococo fueron tratadas con Rifampicina o placebo durante dos djas, en dosis de $10 \mathrm{mg}$. por kilo de peso. La elección de casos para tratar con droga o placebo se efectuó en fonna aleatoria y usando el sistema de doble ciego.

Al tercero y décimo días después de iniciado el tratamiento, se practicó un nuevo frotis faríngeo para detectar el efecto negativizador de la condición de portador del antibiótico y compararlo con lo ocurrido con el placebo.

Durante el proceso de toma de muestra para el primer examen de control, se procedió a interrogar a los individuos subre la ocurrencia de efectos adversos de la droga o del placebo sobre los sistemas respiratorio, digestivo, cardiovascular, nervioso y en la piel. A un grupo de 25 niños se le tomó muestras de sangre para determinación de transaminasas pirúvicas y oxalacéticas con el fin de investigar daño hepático.

\section{RESULTADOS}

En la Tabla N. ${ }^{\circ} 1$ se muestra el resultado bacteríológico de los 2.132 frotis faríngeos realizados. El $12 \%$ de los.niños investigados son portadores de meningococos, no existiendo diferencias significativas en cuanto a edad y sexo.

Al $51 \%$ y $49 \%$ de los portadores se les trató con 
Tabla N." 1

Distribucion de portadores de Meríngococo seguin edad y sexo

\begin{tabular}{|c|c|c|c|c|c|c|c|c|c|}
\hline \multirow{2}{*}{$\begin{array}{l}\text { Edad en } \\
\text { años }\end{array}$} & \multicolumn{3}{|c|}{ FEMENINO } & \multicolumn{3}{|c|}{ MASCULINO } & \multicolumn{3}{|c|}{$T O T A L$} \\
\hline & $\begin{array}{l}\text { V." cutsos } \\
\text { examinados }\end{array}$ & $\begin{array}{l}N^{c} \text { pontis- } \\
\text { dores }\end{array}$ & $\%$ & $\begin{array}{c}\mathrm{N}_{*}^{+1} \text { casos } \\
\text { cxaminados }\end{array}$ & $\begin{array}{l}\text { N." porta- } \\
\text { dores }\end{array}$ & $\%$ & $\begin{array}{l}\text { N. "casos } \\
\text { examinadtos }\end{array}$ & $\begin{array}{l}\text { N."porta- } \\
\text { dores }\end{array}$ & $\%$ \\
\hline $0-4$ & 312 & 32 & 10 & 415 & 41 & 10 & 727 & $7: 3$ & 10 \\
\hline $5-9$ & 270 & 40) & 15 & 286 & 42 & 15 & 556 & 82 & 15 \\
\hline $10-14$ & $3(x)$ & 39 & 13 & 280 & 45 & 16 & 580 & 84 & 14 \\
\hline 15 y más & 20.3 & 15 & 7 & 66 & $b$ & 12 & 269 & 23 & 9 \\
\hline Total & 1.085 & 126 & 12 & 1.047 & $1 ; 36$ & $1: 3$ & 2.132 & 262 & 12 \\
\hline
\end{tabular}

Rifampicina o placebo, respectivamente. (Tabla N. ${ }^{\circ} 2$ ).

A 262 casos identificados como portadures se les administró Rifampicina o placebo, según la técnica descrita precedentemente. A 228 se les efectuó frotis faríngeo de control al tercer clía de tratamiento, y a 96, al décimo día.

De lo anterior se puede deducir que existió $13 \%$ de pérdida para el primer examen de control, $y$ $11 \%$ para el segundo.

En la Tabla N. ${ }^{\circ} 3$ se muestra el resultado del tratamiento. En $92 \%$ de los individuos tratados con Rifampicina (118) y en un $35 \%$ de los tratados con placebo, se encontró frotis faríngeo negativo al tercer día de tratamiento. En $96 \%$ de los casos que se negativizaron con la Rilampicina, el frotis faringeo permanecía negativo al décimo día de tratamiento. (Tabla N."4).

En 40 casos se efectuó determinación de la resistencia del meningococo a la Rifampicina, encontrándose sensible a este antibiótico el $100 \%$ de las cepas exarninadas.

Ninguna de las personas tratadas con Rifampicina o placebo y controladas manifestó signos o síntomas atribuibles a la ingestión de este antibiótico.

\section{COMENTARIO}

La tasa de portadores de meningococo observatda en esta investigación es de $12 \%$, situación que es concordante para este grupo de edad con otras investigaciones realizadas a nivel nacional. ${ }^{1} \mathrm{La}$ eficacia del tratamiento con Rifampicina también es similar a la comunicada por otros antores y publicados en la literatura médica.
Tabla N. ${ }^{\circ} 2$

Tipo de trataniento efectuado.

\begin{tabular}{lcc}
\hline \multicolumn{1}{c}{ Tipo } & N.t & $\%$ \\
\hline Rifampicina & 1.34 & 51 \\
Placebo & 128 & 49 \\
\hline Total & 262 & 100 \\
\hline
\end{tabular}

Tabla N. ${ }^{\circ} 3$

Resultado del Erotis faríngeo efectuado al tercer día de tratamiento

\begin{tabular}{|c|c|c|c|c|c|}
\hline \multirow[t]{2}{*}{ Casos } & \multicolumn{2}{|c|}{ Droga } & \multicolumn{2}{|c|}{ Placebo } & \multirow[t]{2}{*}{ Total } \\
\hline & N." & $\%$ & N." & $\%$ & \\
\hline Negativos & 108 & 92 & 39 & 35 & 147 \\
\hline Positivos & 10 & 8 & 71 & 65 & 81 \\
\hline $\begin{array}{l}\text { Total de } \\
\text { casos }\end{array}$ & 118 & 100 & 110 & $100)$ & 228 \\
\hline
\end{tabular}

Tabla N.*4

Resultada del lrotis faringeo de control efectuado al décimo día de tratamiento en $\$ 6$ portadores que se hacen negativos con rifampicina.

\begin{tabular}{lrr}
\hline Resultactos & N. & $\%$ \\
\hline Negativos & 89 & 93 \\
Portadiores & 7 & 7 \\
\hline Total & 86 & I00 \\
\hline
\end{tabular}


En el presente trabajo, los purtadores tratados con Rifampicina se negativizaron en un $92 \%$, en comparación al $35 \%$ de los tratados con placebo, situación que se mantiene a los diez días de tratamiento.

Se considera que esta droga es altamente efectiva para el tratamiento de los portadores, ya que es un antibiótico de buena tolerancia y sin efectos colaterales.

Por otra parte, la ausencia de resistencia en este estudio de las cepas de meningococo a la Rifampicima, constituye otro antecedente significativo para usar con éxito este antibiótico en el tratamiento de los portadores de neiseria meningitidis. 2.3.4

\section{RESUMEN}

Los problemas presentados por las recientes epidemias de meningitis meningocócica ocuridas en varios países, junto a la importancia de los portadores de meningococo en tales epidemias, $y$ la dificultad para encontrar terapias adecuadas, han hecho necesario investigar nuevas y efectivas tormas de profilaxis.

Los autores investigan la eficacia e inocuidad de la Rifampicina (Rimactan) en 2.132 niños entre I y 18 años de edad que asistian a los jardines infantiles y escuelas básicus de Santiago de Chile. A ellos se les efectuó un trotis taríngeo para inves- tigar la condición de portadores de meningococo. EI I $2 \%$ de ellos fue catalogado como portador de meningococo y tratados con Rifampicina o placebo de acuerdo con una selección aleatoria simple por método de doble ciego. Nuevos frotis fieron tomados a los tercero y décimo dias.

EL 92\% de los individuos tratados con Rifampicina fieron negativos al tercer dia, y el $35 \%$ de los tratados con placebo presentaron igual situación. El $96 \%$ de los individuos tratados con hilampicina que se negativizaron, mantienen esta condición al décimu día de tratamiento.

No se observaron signos o sintomas atribuibles al medicamento. No se observó resistencia de las cepas a la Rifampicina.

\section{REFERENCLAS}

${ }^{l}$ Corey G., Rodriguez H., Aguilar y Greve E. Portadores de Meningococo . Publicación intema, Ministerio de Salud. Anoo 1976.

${ }^{2}$ Bean t. Walter ${ }^{2} t$ al. 'The Effect of Rifampin on the Nasopharyngeal Carriage of Neisseria meningitidis in a Military Population'. The Joumal of Infeccious Diseases, Vol. $124 \mathrm{~N},{ }^{\circ} 1$. July L971.

'Det and Sanders. Etficacy of Rifampin in Treatment of Meningococcal Carriers. New Eng]. S. Med. Vol. 28I, N. 12, 1969.

4Siconen ct al. Chentoprophy lactie une of Ritumpin and Minocycline on Rates of Carriage of Neisseria Meningitidisin Ammy Hecruits in Finland". The Journal of Infeceiuus diseuses, Vol. 137, N. 3. March 1978. 